\title{
Morphostructure of the Ampoule of the Large Papilla of a Duck
}

\author{
N.A. Allanazarova \\ Samarkand State University, Samakand, Uzbekistan
}

\begin{abstract}
Annotation
The principles of morphohistological organization of the ampoule of the large duodenal papilla (duodenum) the duck. Morphometric indicators of the ratio of the cavity of the common bile duct and the pancreatic duct are presented. Morphological details in the structure of the ampoule of the large papilla are currently not fully explained. Therefore, the study of itscomposition, the structure of the internal relief of the duck in connection with the nature of nutrition allows us to solve some questions of theoretical and applied histology. These studies will help to solve the problems of modeling certain biological processes in the future.
\end{abstract}

Keywords: ampoule of the large papilla, duodenum, duodenum, duck, ducts, cavities, liver, pancreas, histology, morphology.

DOI: $10.7176 / J S T R / 6-08-03$

\section{Introduction}

Morphological research forms the foundation of many areas of biological knowledge and makes an important contribution to the development of both practical and theoretical problems of biology. Birds are a well-separated group of animals in evolutionary terms and their digestive system in the process of phylogeny is a pronounced adaptive process. At the same time, it is not functionally related to all external environmental factors, but to feed, its production and assimilation. And some birds have been domesticated by humans and this has also affected their digestive system.

The duodenocholepancreatic zone includes the most complex part of the digestive tract: the duodenum, common bile duct, pancreatic duct (pancreatic duct), large nipple (hepatic-pancreatic ampoule) of the duodenum.

The large papilla of the duodenum is the distal part of the terminal Department of the choledochus (TDCh), or Oddi zone, which includes a system of sphincters that regulate the flow of bile and pancreatic secretions to the duodenum. The large nipple is the distal and most powerful part of the sphincter system of the terminal part of the common bile duct, ductus choledochus, which have a circular structure here. Its length is $3-5 \mathrm{~mm}$.

According to A. I. Edemsky and A.V.Svishchev [1], in 97\% of cases, the large duodenal papilla is a single anatomical formation, in $2.6 \%$ of cases there are two papillae at a distance of 3-5 mm from each other: the upper opens the choledoch, and the lower - the main pancreatic duct. However, most often (65$90 \%$ ) the bile and pancreatic ducts merge to form a common channel. In 16-35\% of cases, there is a separate confluence of the ducts into one mouth, in 4-10\% the ducts open into the large papilla with two independent holes and are separated by a partition. Thus, the location of the large duodenal papilla is variable. In most cases, the large papilla occupies the middle and lower third of the vertical part of the duodenum.

In the available literature, such studies have been conducted on representatives of mammalian animals [2,3,4], on some birds (chickens) [5]; on laboratory mammals [6]; on fish and amphibians [7]. Most of them are the works of the author and his students.

Purpose of research:

To study the morphological structure of the ampoule of the large duodenal papilla in ducks. 


\section{Material and methods of research}

The material for the study was an organ complex of the duodenum of 10 ducks, which includes the end section of the common bile and pancreatic ducts. When performing the work, we used classical methods of research in histology. The material was fixed in $12 \%$ neutral formalin. Filling the material in paraffin is made according to the generally accepted method. Sections were stained with hematoxylin-eosin using the van Gieson technique [8]. A consistent study of the sections gave us the opportunity to trace the mechanism of ampoule formation and trace the microrelief of the mucous membrane throughout its entire length. Measurement of the diameter of the ducts, the thickness of the shells and layers of the ampoule of the large duodenal papilla and its components was performed using an ocular ruler inserted into the eyepiece of a light microscope. The study of successive (serial) histotopographic sections of the duodenum, including the large papilla, in the transverse direction allowed us to establish the features of the structure of this structure.

\section{Results and discussion}

The ampoule of the large papilla of the duodenum of ducks has a rather complex structure. The large papilla is a small convex cone-shaped formation formed in the terminal part of the duodenum. In the thickness of the large papilla of the duodenum is an ampoule formed by the fusion of two bile ducts, as well as the mouth of two pancreatic ducts. The ampulla major duodenal papilla weft is formed by the combination of hepatic and intestinal bile duct from gallbladder-intestinal duct, after their entry into the wall of the small intestine.

On cross sections, you can find that the wall of the bile ducts consists of mucous and muscle membranes. The mucosa of the duct wall consists of epithelial lining, its own and muscle plates. Its own plate consists of loose, unformed connective tissue. In ducks, unlike other species of birds, the mucous membrane of the bile ducts is very well developed and occupies almost half of the lumen of the bile ducts, so that their lumen is relatively small. In this case, the mucous membrane of the bile ducts forms a lot of trough-like depressions and few outgrowths.Under the mucous membrane is a muscle membrane formed by several layers of longitudinally directed bundles of smooth myocytes. The thickness of the muscle shell of the walls of the bile ducts is several times greater than the thickness of the muscle layer of the pancreatic ducts. Initially, the thickness of the muscle wall of the hepatic-intestinal and cystic-intestinal ducts in the proximal, Central and distal parts of the duct is 1.3:0.6:0.7 and 2:1.5: 1.4 microns, respectively (table.1). The diameter of these ducts is 1.5:3.5:4.1 and 1.5:2.7:3 microns, respectively. Outside, the bile ducts are covered with a layer of loose, unformed connective tissue. Before entering the wall of the duodenum, the bile ducts have an oval shape.

But first, the hepatic-intestinal bile duct approaches the outer wall. The muscular layer of the duodenum begins to thicken in the place of dense contact with the hepatic-intestinal bile duct. Then, the intestinal muscle fibers change their orientation from longitudinal to oblique and form a small wedge-shaped outgrowth, which gradually lengthens and begins to surround the duct from the outside. In this case, the hepatic-intestinal bile duct begins to sink into the muscle membrane of the duodenum wall. On the outer surface, it is surrounded by a thin layer of longitudinal duodenal muscle, and on the inner part-a thin layer of circular muscle of the small intestine, which separates the duct from its submucosal layer. After sinking the hepatic-intestinal bile duct into the wall of the duodenum, a vesico-intestinal bile duct appears above its surface, which in the same way is embedded in the wall of the duodenum. First, these two bile ducts are connected to each other by their outer shells, consisting of loose unformed connective tissue, which gradually becomes common to these ducts. After immersion in the intestinal wall, the diameter of the ducts begins to increase in size, reaching 6:5:3 microns, and 2.4:6:4.3 microns, respectively, but the ducts themselves still remain separated by their muscle and mucous membranes (table 2 ). 
Table 1. Morphometric parameters of the duct walls before entering the wall of the duck's duodenum (microns)

\begin{tabular}{|c|c|c|c|c|c|c|c|}
\hline \multirow{3}{*}{$\begin{array}{l}\text { Birdspeciesna } \\
\text { me }\end{array}$} & \multirow{3}{*}{$\begin{array}{l}\text { Muscular wall } \\
\text { and the diameter } \\
\text { of the duct }\end{array}$} & \multicolumn{6}{|c|}{ Ducts before entering the wall 12 p.k. } \\
\hline & & \multicolumn{3}{|c|}{ Duct 1} & \multicolumn{3}{|c|}{ Duct 2} \\
\hline & & 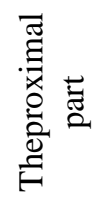 & 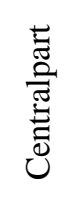 & 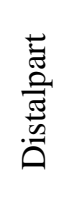 & 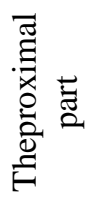 & 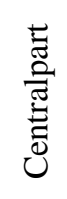 & 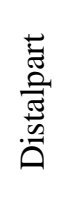 \\
\hline \multirow{2}{*}{ Duck } & $\begin{array}{l}\text { Muscle wall of } \\
\text { the duct }\end{array}$ & 1,3 & 0,6 & 0,7 & 2 & 1,5 & 1,4 \\
\hline & $\begin{array}{c}\text { The diameter of } \\
\text { the duct }\end{array}$ & 1,5 & 3,5 & 4,1 & 1,5 & 2,7 & 3 \\
\hline
\end{tabular}

duct 1 - hepatic-intestinal

duct 2 - vesico-intestinal

The bile ducts begin to form an ampoule. The muscular membrane of the duct walls thicken on the lateral surfaces. Circular muscle layer of the duodenum becomes thinner and gradually disappears, the ampoule is immersed in the submucosal layer of the small intestine. The distal part of the hepatic-intestinal bile duct stretches towards the duodenal cavity, so that this duct changes its shape from oval to trapezoidal. This in turn leads to a change in the shape of the ampoule itself.

As it sinks into the intestinal wall, the ampoule of the large papilla of the duodenum of ducks increases in size. The diameter of the ampoule of the large papilla of the duodenum of ducks is 5.7 microns in the proximal part, 3.4 microns in the Central part and 2 microns in the distal part (table.2). The distal part of the ampoule forms a small mouth opening into the intestinal cavity. The diameter of the mouth of the ampoule is 1.5 microns, the length of the ampoule is 9 microns, and its area is $124 \mathrm{cu}$. The entire cavity of the ampoule, especially its distal part, is permeated by outgrowths of the mucous membrane. Between them are depressions that form small cruciform passages in the submucosal layer.

Then the septum that divides the ampoule into two large chambers is thinned and the vesico-intestinal bile duct is pressed into its distal part and begins to sink into the cavity of the hepatic-intestinal duct. The side walls of the cystic-intestinal duct begin to diverge in different directions and then merge with the side walls of the ampoule. As a result, the size of the ampoule wall increases. In it, the epithelium is embedded in the thickness of the ampoule wall, and wall-mounted common intestinal glands are formed, which are of the type of simple tubular glands. The ampoule has an expanded part at the base and a more narrowed distal part.

The wall of the ampoule of the large papilla of the duodenum of ducks consists of mucous and muscle membranes. The mucosa consists of epithelial cells, the nuclei of which are located in the basal part of the cells. At the same time, the cores are usually located at the same level. The mucous membrane of the ampoule forms a lot of vypyachivanii in its own plate. Also, the mucous membrane of the ampoule forms outgrowths (dampers) that permeate the cavity of the ampoule itself. Outgrowths are often irregular in shape, with uneven surfaces, often branching and joining with other outgrowths to form anastomoses. Between these connections are holes or chambers that have different sizes and shapes, due to varying thickness and shape of the dampers themselves. The valves of the ampoule of the large papilla of the duodenum of ducks differ in a variety of shapes and complexity of their location. For example, the distal portion of the ampoule flap become less curvy, more pointed and, unlike the flaps of the proximal ampoules, their tops directed towards the location of the mouth, and not in the Central part of the ampoule. The tops of the dampers do not close with each other, but hang freely at the mouth of the ampoule. 
Table 2. Morphometric parameters of ducts in the wall and ampoules of the large papilla of the duodenum of a duck (microns)

\begin{tabular}{|c|c|c|c|c|c|c|c|c|c|c|}
\hline \multirow{3}{*}{$\begin{array}{c}\text { Bird species } \\
\text { name }\end{array}$} & \multirow{3}{*}{$\begin{array}{l}\text { Muscular wall } \\
\text { and the } \\
\text { diameter of } \\
\text { the duct }\end{array}$} & \multicolumn{6}{|c|}{ Ducts in the wall 12 p.k. } & \multirow{2}{*}{\multicolumn{3}{|c|}{$\begin{array}{c}\text { Ampoule of the } \\
\text { large papilla } 12 \\
\text { p.k. }\end{array}$}} \\
\hline & & \multicolumn{3}{|c|}{ Duct 1} & \multicolumn{3}{|c|}{ Duct 2} & & & \\
\hline & & 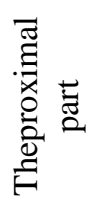 & 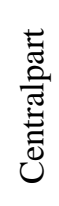 & 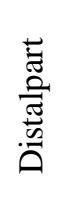 & 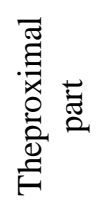 & 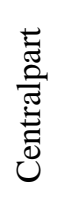 & 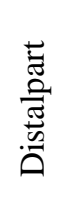 & 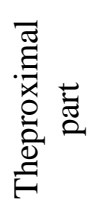 & 壭 & 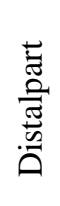 \\
\hline \multirow{2}{*}{ Duck } & $\begin{array}{l}\text { Muscle wall } \\
\text { of the duct }\end{array}$ & 0,9 & 0,6 & 0,6 & 0,7 & 0,7 & 0,6 & 0,7 & 1,4 & 0,6 \\
\hline & $\begin{array}{l}\text { The diameter } \\
\text { of the duct }\end{array}$ & 6 & 5 & 3 & 2,4 & 6 & 4,3 & 5,7 & 3,4 & 2 \\
\hline
\end{tabular}

The diameter of the mouth of the ampoule is 12 p.k. $-1,5$

\author{
Area of the ampoule - 124 duct 1 - hepatic-intestinal \\ The length of the ampoule $-9 \quad$ duct 2 - vesico-intestinal
}

We found that the largest size of the flap is reached in the distal part of the ampoule. Functionally, these dampers prevent the reverse flow of intestinal contents into the ampoule. Crypts and tubular glands lie deep in their own plate of the wall of the ampoule of the large papilla. Under the mucous membrane of the ampoule is located the muscle membrane, which consists of smooth muscle tissue. Its thickness decreases in the distal direction. Thus, ampulla major duodenal papilla weft is thicker than the submucosa of the duodenum. Almost all sides, except the proximal part of the ampoule is surrounded by a layer of connective tissue. The base of the ampoule borders on the inner circular layer of the muscular wall of the duodenum. The side surface of the ampoule is surrounded by the intestinal mucosa. By inserting the epithelium of the small intestine into the thickness of its wall, wall-mounted common intestinal glands and duodenal glands are formed. The common intestinal glands are located at the base of the mucous membrane. Their ducts open between the bases of the villi of the duodenum. Among the main mass of cells of a single-layer cylindrical epithelium lining the lumen of such a gland, there are many gobletshaped cells. Cylindrical cells are cambial, and goblet-shaped cells are involved in the production of a secret that moisturizes the lumen of the small intestine. The duodenal glands lie in several rows in the submucosa of the intestine. Their wall is also constructed from a single-layer cylindrical epithelium. Their excretory ducts exit between the common intestinal glands. From the protrusions of the mucous membrane into the lumen of the intestine, intestinal villi are formed. In the area where the ampoule is located, there are almost no ampoules. The villi of the duodenum of ducks have a leaf-like shape and a spiral arrangement. Each villi is covered with a single-layer cylindrical edged epithelium, in which there are also goblet cells.

Under this ampoule, the pancreatic ducts begin to open, which alternately form two independent mouths and also flow into the cavity of the duodenum of ducks.

\title{
Conclusions
}

Thus, in a duck, the diameter of the hepatic and pancreatic ducts in the wall of the duodenum almost doubles. The diameter of the hepatic duct of a duck outside the duodenum wall in the proximal, Central and distal parts of the duct is 1.5:3.5:4.1 microns, after entering the duodenum wall is 6:5:3 microns. Changes in the size of the cystic duct are also observed. (outside the wall-1.5:2.7:3, in the wall-2.4:6:4.3 microns). The results obtained are a contribution to the study of the structure and structure of the internal relief of the ampoule of the large papilla in birds with different conditions of existence and the nature of nutrition. This, in turn, will solve some questions of theoretical and applied histology. In the future, these 
studies will help solve the problems of modeling certain biological processes, in particular, the regulatory systems of the body.

\section{REFERENCES:}

[1]. Edemsky A. I., Svishchev A.V. Anatomical and stereological characteristics of the mucous membrane of the large duodenal papilla / / Archive of anat.gistol. andembr. 1986, N. 3, pp. 6166.

[2]. Dekhkanov T. D., Akhmedov A. Comparative morphology of the hepatic-pancreatic ampoule in mammalian animals with different types of nutrition. // Problems of biology and medicine, 2002, N. 4. pp. 34-36.

[3]. Allanazarova N. A. Adelova M. R. Some regularities of morphological structure of hepaticpancreatic ampoules of animals Collection of articles VIII international scientific conference "Modern trends in science and technology" Dedicated to the 25th anniversary of the Korean scientific and technical societies of SNG countries (Uzbekistan, Kazakhstan, Russia) on November 18-19 2016, Tashkent, p. 307-311

[4]. Allanazarova N. A. Using data on the morphology of the liver-pancreatic ampoule in some mammals in medicine /Materials of the 2nd Republican scientific-practical seminar "Current and prospective problems of medicine in Uzbekistan". Abstracts of reports, 2010. - p. 98

[5]. Allanazarova N. A., Shakirova M. I. Features of the structure of the liver-pancreatic ampoule duodenum in chicken. Scientific works of the Moscow medical Academy named after M. M. Sechenov. //Problems of ecology, health, formation and Parasitology, Moscow, 2005, pp. 183189

[6]. Sadullayeva M. S. Morphology of the hepatic-pancreatic ampoule in some mammals. //Materials of the XLIV International scientific student conference. - Novosibirsk, 2006. - p. 98-100

[7]. Comparative histological studies of the formation of the fater's papilla in some amphibians and fish Man-Nature-Society: theory and practice of life safety, ecology and valeology. Issue 4 (11): Collection of scientific papers. - Simferopol: RIO KIPU, 2018, p. 47-50

[8]. Fundamentals of histology and histological techniques / edited by prof. E.G.Eliseev M.: "Medicine", 1967, 268p. 